\title{
Ehrhart $f^{*}$-coefficients of polytopal complexes are non-negative integers
}

\author{
Felix Breuer* \\ Department of Mathematics \\ San Francisco State University \\ California, U.S.A. \\ felix@fbreuer.de
}

Submitted: Mar 7, 2012; Accepted: Oct 16, 2012; Published: Nov 1, 2012

Mathematics Subject Classifications: 52B20, 52B70, 05A10, 05A15, 05E45, 11C08

\begin{abstract}
The Ehrhart polynomial $L_{P}$ of an integral polytope $P$ counts the number of integer points in integral dilates of $P$. Ehrhart polynomials of polytopes are often described in terms of their Ehrhart $h^{*}$-vector (aka Ehrhart $\delta$-vector), which is the vector of coefficients of $L_{P}$ with respect to a certain binomial basis and which coincides with the $h$-vector of a regular unimodular triangulation of $P$ (if one exists). One important result by Stanley about $h^{*}$-vectors of polytopes is that their entries are always non-negative. However, recent combinatorial applications of Ehrhart theory give rise to polytopal complexes with $h^{*}$-vectors that have negative entries.

In this article we introduce the Ehrhart $f^{*}$-vector of polytopes or, more generally, of polytopal complexes $K$. These are again coefficient vectors of $L_{K}$ with respect to a certain binomial basis of the space of polynomials and they have the property that the $f^{*}$-vector of a unimodular simplicial complex coincides with its $f$-vector. The main result of this article is a counting interpretation for the $f^{*}$-coefficients which implies that $f^{*}$-coefficients of integral polytopal complexes are always non-negative integers. This holds even if the polytopal complex does not have a unimodular triangulation and if its $h^{*}$-vector does have negative entries. Our main technical tool is a new partition of the set of lattice points in a simplicial cone into discrete cones. Further results include a complete characterization of Ehrhart polynomials of integral partial polytopal complexes and a non-negativity theorem for the $f^{*}$-vectors of rational polytopal complexes.
\end{abstract}

Keywords: Ehrhart theory, $f^{*}$-vector, $h^{*}$-vector, Ehrhart polynomial, counting interpretation, non-negativity, partial polytopal complex, simplicial complex, discrete cone, affine semigroup.

${ }^{*}$ The author was supported by the DFG (Deutsche Forschungsgemeinschaft) grant BR 4251/1-1. 


\section{Introduction}

For any set $X \subset \mathbb{R}^{n}$ the Ehrhart function $L_{X}(k)=\#\left(\mathbb{Z}^{n} \cap k X\right)$ counts the number of lattice points in the $k$-th dilate of $X$ for $1 \leqslant k \in \mathbb{Z}$. Ehrhart's theorem states that if $P$ is a lattice polytope then $L_{P}(k)$ is a polynomial in $k$ and, by induction, the same holds for polytopal complexes with integral vertices $[1,10,11]$.

Recently, a number of articles have appeared that realize various combinatorial counting polynomials as Ehrhart functions of suitable polytopal complexes and then apply results from Ehrhart theory to prove theorems about these combinatorial functions [3, 4, $8,15]$. In particular, it is possible to obtain bounds on the coefficients of these polynomials in this way [6]. For this purpose, the coefficients with respect to the monomial basis are not always easiest to work with. There are other bases of polynomial space that give rise to coefficient vectors such as the $h^{*}$ - and $f^{*}$-vectors that are more amenable to analysis. These are defined as follows.

Let $p(k)$ be a polynomial in $k$ of degree at most $d$. Then there exist coefficients $h_{i}^{*}$ and $f_{i}^{*}$ for $i=0, \ldots, d$ such that

$$
p(k)=\sum_{i=0}^{d} h_{i}^{*}\left(\begin{array}{c}
k+d-i \\
d
\end{array}\right)=\sum_{i=0}^{d} f_{i}^{*}\left(\begin{array}{c}
k-1 \\
i
\end{array}\right) .
$$

The coefficients $h_{i}^{*}$ and $f_{i}^{*}$ depend both on $p$ and on $d$, so we will sometimes write $h_{i}^{*}(p, d)$ and $f_{i}^{*}(p, d)$ to make this dependency explicit. The vectors $\left(h_{0}^{*}, \ldots, h_{d}^{*}\right)$ and $\left(f_{0}^{*}, \ldots, f_{d}^{*}\right)$ are called the $h^{*}$ - and $f^{*}$-vectors of $p$ and their entries are the $h^{*}$ - and $f^{*}$-coefficients of $p$, respectively. Note that the $h^{*}$-vector also goes by the name of the Ehrhart $\delta$-vector [18]. Whenever we refer to the $h^{*}$ - or $f^{*}$-vector of an integral polytope or polytopal complex $P$, we mean the $h^{*}$ - or $f^{*}$-vector of its Ehrhart polynomial $L_{P}$. For more details on these vectors and, most importantly, the motivation for defining them we refer the reader to Section 2.3.

One famous result about $h^{*}$-vectors is Stanley's theorem which asserts that the $h^{*}$ coefficients of the Ehrhart polynomial of an integral polytope are always non-negative integers [17]. Behind this theorem lies a beautiful interpretation, due to Ehrhart, of the $h^{*}$-coefficients of the Ehrhart polynomial of a simplex $\Delta$ as counting lattice points at various heights in the fundamental parallelepiped of the cone over the homogenization of $\Delta[10,11]$.

While $h^{*}$-vectors of integral polytopes are always non-negative, $h^{*}$-vectors of integral polytopal complexes may well have negative entries. Moreover, polytopal complexes with negative $h^{*}$-coefficients appear in natural combinatorial applications. For example, coloring complexes of uniform hypergraphs can have negative $h^{*}$-coefficients. Their $f^{*}$-vector, however, is always non-negative. See Section 2.6 and [7] for details.

Thus, the question arises whether this is always true: Do polytopal complexes always have a non-negative integral $f^{*}$-vector? The purpose of this article is to give a positive answer to this question.

Our main result is a counting interpretation of the $f^{*}$-vector of a simplex $\Delta$, in the spirit of the classic counting interpretation of the $h^{*}$-vector of a simplex. Given a relatively 
open lattice simplex $\Delta$, the $f^{*}$-vector counts the number of so-called atomic lattice points at different heights in the fundamental simplex of the cone over the homogenization of $\Delta$. More precisely:

Theorem 1. Let $\Delta \subset \mathbb{R}^{n}$ be an open lattice simplex, let $d^{\prime} \geqslant d=\operatorname{dim}(\Delta)$ and let $f^{*}\left(L_{\Delta}, d^{\prime}\right)=\left(f_{0}^{*}, \ldots, f_{d^{\prime}}^{*}\right)$. Then $f_{i}^{*}$ counts the number of atomic lattice points in the half-open fundamental simplex of $\operatorname{cone}_{\mathbb{R}}(\Delta \times\{1\})$ at level $i+1$.

The definitions of the fundamental simplex, atomic lattice points and their level are given in Section 3. An open lattice simplex is the relative interior of a lattice simplex.

From this counting interpretation we can immediately obtain a complete characterization of the $f^{*}$-vectors of integral partial polytopal complexes. Here, an integral partial polytopal complex is any set that can be written as the disjoint union of relatively open polytopes with integral vertices.

Theorem 2. A vector is the $f^{*}$-vector of some integral partial polytopal complex if and only if it is integral and non-negative.

In particular, this gives us the desired non-negativity result for $f^{*}$-vectors of polytopal complexes.

Theorem 3. Every integral polytopal complex, and in particular every lattice polytope, has a non-negative integral $f^{*}$-vector.

The crucial point here is that the $f^{*}$-vector is non-negative and integral even if the complex does not have unimodular triangulation and even if its $h^{*}$-vector has negative entries. Note that non-negativity of the $f^{*}$-vector follows automatically if the complex has a unimodular triangulation or if the $h^{*}$-vector is non-negative. This means that Theorem 3 gives a new result only if the complex in question is non-convex and does not have a non-negative $h^{*}$-vector. But, as we already mentioned, there are non-convex polytopal complexes with negative $h^{*}$-coefficients that do appear in practical applications.

The key technical ingredient that goes into the above counting interpretation is the following partition of the set of lattice points in a simplicial cone into "discrete cones".

Theorem 4. Let $v_{1}, \ldots, v_{d}$ be linearly independent integer vectors in $\mathbb{Z}^{n}$ for $n \geqslant d$. Then

$$
\operatorname{relint}\left(\operatorname{cone}_{\mathbb{R}}\left(v_{1}, \ldots, v_{d}\right)\right) \cap \mathbb{Z}^{n}=\bigcup_{z \text { atomic }} z+\operatorname{cone}_{\mathbb{Z}}\left(v_{1}, \ldots, v_{\operatorname{lev}(z)}\right),
$$

where the union ranges over all atomic lattice points in the half-open fundamental simplex of cone $_{\mathbb{R}}\left(v_{1}, \ldots, v_{d}\right)$ and this union is disjoint.

Here $\operatorname{lev}(z)$ denote the level of $z$ and $\operatorname{cone}_{\mathbb{R}}\left(v_{1}, \ldots, v_{d}\right)$ refers to all non-negative linear combinations of the $v_{i}$ whereas cone $_{\mathbb{Z}}\left(v_{1}, \ldots, v_{d}\right)$ refers to all non-negative, integral linear combinations of the $v_{i}$, i.e., the affine semigroup generated by the $v_{i}$. Again, we refer to Sections 2 and 3 for details.

Theorem 4 is much more general then necessary for Theorems 1, 2 and 3 and is the main technical result of this article. In particular, Theorem 4 can be used to obtain a counting interpretation and a non-negativity theorem in the rational case. 
Theorem 5. Let $\Delta \subset \mathbb{R}^{n}$ be an open rational simplex, let $d^{\prime} \geqslant d=\operatorname{dim}(\Delta)$ and $m$ be a positive integer such that $m \Delta$ is integral. There exist polynomials $p_{0}, \ldots, p_{m-1}$ such that for all integers $k \geqslant 0$ and $0 \leqslant l<m$ with $(k, l) \neq(0,0)$ the Ehrhart function of $\Delta$ satisfies $L_{\Delta}(k m+l)=p_{l}(k)$. Then for all $0 \leqslant i \leqslant d$ and all $0 \leqslant l<m$ the $f^{*}$-coefficient $f_{i}^{*}\left(p_{l}, d\right)$ counts the number of atomic lattice points $z$ in the half-open fundamental simplex of cone $_{\mathbb{R}}(\Delta \times\{m\})$ at level $i+1$ with $z_{n+1}=i m+l+1$.

This counting interpretation yields a non-negativity theorem for the $f^{*}$-vector just as in the integral case. The $f^{*}$-vector of a rational polytopal complex is the vector of all numbers $f_{i}^{*}\left(p_{l}, d\right)$ for $i=0, \ldots, d$ and $0 \leqslant l<m$, see Section 5 for details.

Theorem 6. Any rational partial polytopal complex has a non-negative integral $f^{*}$-vector.

Interestingly, there is another variant of Theorem 5 that expresses the Ehrhart function of a rational simplex in terms of restricted partition functions. For our purposes the restricted partition function $p_{m_{1}, \ldots, m_{d}}(k)$ is given by

$$
p_{m_{1}, \ldots, m_{d}}(k)=\#\left\{\left(\lambda_{1}, \ldots, \lambda_{d}\right) \mid 0 \leqslant \lambda_{i} \in \mathbb{Z}, \sum_{i=1}^{d} \lambda_{i} m_{i}=k\right\},
$$

see Section 5 for details. Then Theorem 4 allows us to write the Ehrhart function of a rational simplex in terms of restricted partition functions in the following way.

Theorem 7. Let $\Delta \subset \mathbb{R}^{n}$ be an open lattice simplex with vertices $v_{1}, \ldots, v_{d+1}$ and let $m_{1}, \ldots, m_{d+1}$ be minimal positive integers such that $m_{i} v_{i}$ is integral for all $1 \leqslant i \leqslant d+1$. Then

$$
L_{\Delta}(k)=\sum_{i=0}^{d} \sum_{s=0}^{S} c_{i, s} \cdot p_{m_{1}, \ldots, m_{i+1}}(k-s)
$$

for all $1 \leqslant k$ where $S=\sum_{i=1}^{d+1} m_{i}$ and $c_{i, s}$ denotes the number of atomic lattice points $z$ at level $i+1$ in the fundamental simplex of $\operatorname{cone}_{\mathbb{R}}\left(a_{1}, \ldots, a_{d+1}\right)$ with $z_{n+1}=s$. Here, $a_{i}=\left(m_{i} v_{i, 1}, \ldots, m_{i} v_{i, n}, m_{i}\right)$ for all $i=1, \ldots, d+1$.

This paper is organized as follows. In Section 2 we give some preliminary definitions, sketch a classic proof of the non-negativity of $h^{*}$-vectors for polytopes and give an example of a natural simplicial complex with a negative $h^{*}$-vector. In Section 3 we present the partition of the set of lattice points in an open simplicial cone into discrete subcones, which is the main technical result of this article. In Section 4 we use this partition result to give a counting interpretation of the $f^{*}$-coefficients of a simplex, prove the non-negativity of the $f^{*}$-vector and give a complete characterization of the Ehrhart polynomials of integral partial polytopal complexes. Up to this point we have mainly worked with integral polytopes, to make the ideas behind the construction more transparent. However, most of our results apply to the rational case as well. In Section 5 we introduce $f^{*}$-vectors of rational polytopes, give a counting interpretation, prove the non-negativity of the $f^{*}$-vectors of rational partial polytopal complexes and relate Ehrhart functions of rational simplices to restricted partition functions. 


\section{Preliminaries}

Note: A comprehensive definition of all notions from polytope theory, Ehrhart theory or generating function theory that we make use of is out of scope of this article. For any undefined terms we refer the reader to [1, 16, 21].

\subsection{Geometry}

A polytope is the convex hull of finitely many points. A supporting hyperplane of a polytope $P$ is a hyperplane such that $P$ is contained in one of the two corresponding closed half-spaces. A face of $P$ is the intersection of a supporting hyperplane with $P$. By convention $P$ is a face of itself as well. The dimension of $P$ is the dimension of its affine hull. The faces of dimension 0 are called vertices.

A polytope is integral if all its vertices are elements of the integer lattice $\mathbb{Z}^{n}$, where $n$ is the dimension of the ambient space. Integral polytopes are also called lattice polytopes. Two polytopes $P, Q$ are lattice equivalent if there is an affine isomorphism $\phi$ of the ambient space with $\phi(P)=Q$ that induces a bijection on the integer lattice $\mathbb{Z}^{n}$.

The relative interior of a polytope $P$ is the interior of $P$ taken with respect to its affine hull. We also use the term open polytope to refer to the relative interior of a polytope. When we speak of the faces of an open polytope, we mean the faces of its closure. Every polytope is the disjoint union of the relative interiors of its faces.

A simplex is the convex hull of finitely many affinely independent points. A simplex of dimension $d$ has exactly $d+1$ vertices. The standard simplex $\Delta^{d}$ of dimension $d$ is the convex hull of $d+1$ standard unit vectors. An integral simplex is unimodular if it is lattice equivalent to a standard simplex.

A polytopal complex is a finite set of polytopes $K$ with the following two properties: 1) If $P \in K$ and $Q$ is a face of $P$, then $Q \in K$. 2) If $P, Q \in K$, then $P \cap Q \in K$ and $P \cap Q$ is a face of both $P$ and $Q$. The elements of $K$ are also called faces of $K$. The dimension of $K$ is the maximum dimension of any face of $K$. The support of $K$ is the union of all polytopes in $K$. A polytopal complex is integral if all of its faces are integral.

A simplicial complex is a polytopal complex whose faces are simplices. A triangulation of a set $X \subset \mathbb{R}^{n}$ is a simplicial complex whose support is $X$. A simplicial complex is unimodular if all of its faces are unimodular. Note that not all integral polytopes, not even all integral simplices, have a unimodular triangulation.

The $f$-vector of a $d$-dimensional simplicial complex $K$ is the vector $\left(f_{0}, f_{1}, \ldots, f_{d}\right)$ where $f_{i}$ is the number of $i$-dimensional faces of $K$. The h-vector of $K$ is the vector $\left(h_{0}, \ldots, h_{d+1}\right)$ defined in terms of the $f$-vector via

$$
h_{k}=\sum_{i=0}^{k}(-1)^{k-i}\left(\begin{array}{l}
d-i \\
d-k
\end{array}\right) f_{i-1}
$$

for $k=0, \ldots, d+1$ where $f_{-1}:=1$. Note that the $h$-vector has one more entry than the $f$-vector but $h_{0}=1$ is fixed. 


\subsection{Ehrhart theory}

As mentioned in the introduction, our point of departure is Ehrhart's theorem, which states that for any integral polytope $P \in \mathbb{R}^{d}$ there exists a polynomial $L_{P}(k)$ such that

$$
\#\left(\mathbb{Z}^{d} \cap k P\right)=L_{P}(k)
$$

for all $1 \leqslant k \in \mathbb{Z}$.

It is straightforward to see that Ehrhart's theorem carries over to polytopal complexes. However, many applications go one step further and work with "partial" polytopal complexes instead, where some faces are missing. In particular, inside-out polytopes are examples of half-open polytopal complexes that are widely used in combinatorial applications of Ehrhart theory $[3,4,6]$. Let us now make precise what we mean by "partial" in this context.

As defined in the previous section, a (relatively) open polytope is the relative interior of a polytope. The vertices, faces and facets of an open polytope are defined to be the vertices, faces and facets of its closure. Note that thus the vertices of an open polytope are not contained in the open polytope. An open polytope is called integral if all its vertices are integral.

For any polytopal complex $K$, the support of $K$ is the disjoint union of the relative interiors of all faces of $K$. This motivates the following definition: A partial polytopal complex is a disjoint union of open polytopes. The difference between a polytopal complex and a partial polytopal complex is therefore simply that some of the relatively open faces of the polytopal complex (that would need to be included because a polytopal complex has to be closed under passing to faces) have been removed.

Two important special cases of partial polytopal complexes are the following.

A "half-open" polytope is a set of the form $P \backslash \bigcup_{i=1}^{l} \sigma_{i}$ where $P$ is a polytope and the $\sigma_{i}$ are faces of $P$. Every half-open polytope is the support of some partial polytopal complex. The half-open simplices that we will meet in the next section are examples of this.

A relative simplicial complex is a set of simplices of the form $K \backslash K^{\prime}$ where $K$ is a simplicial complex and $K^{\prime}$ is a subcomplex of $K$. Relative simplicial complexes can be written as partial polytopal complexes. They appear, for example, in Steingrímsson's construction of the coloring complex [20]. Relative polytopal complexes can be defined similarly and again they can be realized as partial polytopal complexes. Inside-out polytopes are examples of relative polytopal complexes [5].

\section{$2.3 \quad f^{*}$ - and $h^{*}$-vectors}

Let us denote by $\Delta_{i}^{d}$ the $d$-dimensional standard simplex with $i$ open facets, i.e.,

$$
\Delta_{i}^{d}:=\left\{x \in \mathbb{R}^{d+1} \mid \sum_{j=0}^{d} x_{j}=1, x_{j}>0 \text { for } 0 \leqslant j<i, x_{j} \geqslant 0 \text { for } i \leqslant j \leqslant d\right\} .
$$

THE ELECTRONiC JOURNAL OF COMBINATORICS 19(4) (2012), \#P16 
It turns out that $L_{\Delta_{i}^{d}}(k)=\left(\begin{array}{c}k+d-i \\ d\end{array}\right)$ for $i=0, \ldots, d+1$ and in particular $L_{\Delta_{d+1}^{d}}(k)=$ $\left(\begin{array}{c}k-1 \\ d\end{array}\right)$ where $\Delta_{d+1}^{d}=$ relint $\Delta^{d}$ is the relative interior of the standard $d$-dimensional simplex. To see this, one can use an argument similar to the one given in [1, Section 2.3]. These observations have the following immediate consequences for a $d$-dimensional integral polytopal complex $C$.

1. If $C$ has a unimodular triangulation $K$, then $C$ can be written as a disjoint union of relatively open unimodular simplices $\Delta_{i+1}^{i}$ of varying dimension $i=0, \ldots, d$. Thus

$$
L_{C}(k)=\sum_{i=0}^{d} f_{i}^{*}\left(\begin{array}{c}
k-1 \\
i
\end{array}\right)
$$

where the coefficients $f_{i}^{*}$ count the number of $i$-dimensional relatively open unimodular simplices appearing in the disjoint union. In this case the $f$-vector of the simplicial complex $K$ coincides with the vector of coefficients $f_{i}^{*}$, which explains the name.

2. If $C$ has a unimodular triangulation $K$ that can be written as a disjoint union of unimodular half-open simplices $\Delta_{i}^{d}, i=0, \ldots, d$ of fixed dimension $d$, then

$$
L_{C}(k)=\sum_{i=0}^{d} h_{i}^{*}\left(\begin{array}{c}
k+d-i \\
d
\end{array}\right)
$$

where the coefficients $h_{i}^{*}$ count the number of $i$-dimensional relatively open unimodular simplices appearing in the disjoint union. In particular, if $K$ is a shellable ${ }^{1}$ complex that is a topological ball then the $h$-vector of $K$ coincides with the vector of coefficients $h_{i}^{*}$, which explains the name. Note that if $K$ is not a topological ball, then $h_{d+1}$ is non-zero in general and the $h^{*}$ - and $h$-vectors may differ.

If $C$ does not have a unimodular triangulation, we can still define the $h^{*}$ - and $f^{*}$ vectors of $C$. In fact, we can define $h^{*}$ - and $f^{*}$-vectors for arbitrary polynomials by proceeding as sketched in the introduction.

For any integer $i$,

$$
\left(\begin{array}{c}
k \\
i
\end{array}\right)=\frac{k \cdot(k-1) \cdot \ldots \cdot(k-i+1)}{i !}
$$

is a polynomial in $k$ of degree $i$. Moreover, both

$$
\left\{\left(\begin{array}{c}
k-1 \\
i
\end{array}\right) \mid i=0, \ldots, d\right\} \text { and }\left\{\left(\begin{array}{c}
k+d-i \\
d
\end{array}\right) \mid i=0, \ldots, d\right\}
$$

form bases of the vector space of polynomials in $k$ of degree at most $d$. Thus, for any non-negative integer $d$ and any polynomial $p(k)$ of degree at most $d$ we can define vectors

\footnotetext{
${ }^{1}$ See [21] for the definition of shellable.
} 


$$
\begin{aligned}
f^{*}(p, d)=\left(f_{0}^{*}, \ldots, f_{d}^{*}\right) \text { and } h^{*}(p, d) & =\left(h_{0}^{*}, \ldots, h_{d}^{*}\right) \text { by } \\
p(k) & =\sum_{i=0}^{d} f_{i}^{*}\left(\begin{array}{c}
k-1 \\
i
\end{array}\right) \\
p(k) & =\sum_{i=0}^{d} h_{i}^{*}\left(\begin{array}{c}
k+d-i \\
d
\end{array}\right) .
\end{aligned}
$$

We call $f^{*}(p, d)$ the $f^{*}$-vector of $p$ and the numbers $f_{i}^{*}$ the $f^{*}$-coefficients of $p$. Similarly, we call $h^{*}(p, d)$ the $h^{*}$-vector of $p$ and the numbers $h_{i}^{*}$ the $h^{*}$-coefficients of $p$.

At this point, it important to call attention to the following subtlety: $h^{*}$ depends on the choice of $d$, whereas $f^{*}$ does not. More precisely, the $f^{*}$-vector has the following property. Let $p$ be any polynomial and let $d_{1}, d_{2} \geqslant \operatorname{deg}(p)$ be any two integers. Then $f_{i}^{*}\left(p, d_{1}\right)=f_{i}^{*}\left(p, d_{2}\right)$ for all $0 \leqslant i \leqslant \min \left(d_{1}, d_{2}\right)$. This statement is false for $h^{*}$. Despite this difference, we will suppress $p$ and $d$ in our notation for both $f^{*}$ and $h^{*}$ whenever it is clear from context which $p$ and $d$ are meant.

Now that we have defined the $f^{*}$ - and $h^{*}$-vectors of polynomials, we can define $f^{*}$ and $h^{*}$-vectors of polytopes (and more generally polytopal complexes) via the Ehrhart function.

Let $K$ denote a polytopal complex. Then the $f^{*}$ - and $h^{*}$-vectors of $K$ are, respectively, defined by

$$
\begin{aligned}
& f^{*}(K, d)=f^{*}\left(L_{K}, d\right) \\
& h^{*}(K, d)=h^{*}\left(L_{K}, d\right),
\end{aligned}
$$

where $d \geqslant \operatorname{dim}(K)$.

If we do not specify $d$ explicitly, it is understood that $d=\operatorname{dim}(K)$, that is, $f^{*}(K)=$ $f^{*}(K, \operatorname{dim}(K))$ and $h^{*}(K)=h^{*}(K, \operatorname{dim}(K))$.

\subsection{Generating function point of view}

Classically, the $h^{*}$-vector is defined in terms of generating functions.

Proposition 8 (c.f. Lemma 3.14 in [1]). If $p$ is a polynomial of degree at most $d$, then

$$
\frac{h_{0}^{*} z^{0}+\ldots+h_{d}^{*} z^{d}}{(1-z)^{d+1}}=\sum_{k \geqslant 0} p(k) z^{k} .
$$

A similar statement can be made about the $f^{*}$-vector.

Proposition 9. If $p$ is a polynomial of degree at most $d$, then

$$
\frac{f_{0}^{*} z^{1}}{(1-z)^{1}}+\cdots+\frac{f_{d}^{*} z^{d+1}}{(1-z)^{d+1}}=\sum_{k \geqslant 0} p(k) z^{k} .
$$


Proof. The coefficient of $z^{k}$ in the Laurent expansion of $\frac{1}{(1-z)^{j+1}}$ is precisely $L_{\Delta^{j}}(k)=$ $\left(\begin{array}{c}k+j \\ j\end{array}\right)$, the number of lattice points in the $k$-th dilate of a $j$-dimensional unimodular simplex. Thus

$$
\frac{z^{j+1}}{(1-z)^{j+1}}=\sum_{k \geqslant 0}\left(\begin{array}{c}
k-1 \\
j
\end{array}\right) z^{k}
$$

which yields the desired identity.

Corollary 10. The $f^{*}$ - and $h^{*}$-vectors of a polynomial $p$ satisfy

$$
h_{0}^{*} z^{0}+\ldots+h_{d}^{*} z^{d}=\sum_{j=0}^{d} f_{j}^{*} z^{j+1}(1-z)^{d-j} .
$$

\subsection{Counting interpretation for the $h^{*}$-vector}

Given linearly independent integer vectors $a_{1}, \ldots, a_{n} \subset \mathbb{Z}^{d}$ we define the cone over the $a_{i}$ by

$$
\operatorname{cone}_{\mathbb{R}}\left(a_{1}, \ldots, a_{n}\right)=\left\{x \in \mathbb{R}^{d} \mid x=\sum_{i=1}^{n} \lambda_{i} a_{i}, 0 \leqslant \lambda_{i} \in \mathbb{R}\right\} .
$$

Instead of allowing real coefficients $\lambda_{i}$, we can also restrict ourselves to integral coefficients. In this way, we obtain the discrete cone over the $a_{i}$ which is

$$
\operatorname{cone}_{\mathbb{Z}}\left(a_{1}, \ldots, a_{n}\right)=\left\{x \in \mathbb{R}^{d} \mid x=\sum_{i=1}^{n} \lambda_{i} a_{i}, 0 \leqslant \lambda_{i} \in \mathbb{Z}\right\} .
$$

The discrete cone is the affine semigroup generated by the $a_{i}$.

The fundamental parallelepiped $\Pi\left(a_{1}, \ldots, a_{n}\right)$ of the cone is

$$
\Pi\left(a_{1}, \ldots, a_{n}\right)=\left\{x \in \mathbb{R}^{d} \mid x=\sum_{i=1}^{n} \lambda_{i} a_{i}, 0 \leqslant \lambda_{i}<1, \lambda_{i} \in \mathbb{R}\right\} .
$$

The crucial property of the fundamental parallelepiped is that it tiles the cone. That is, the cone can be written as the disjoint union of integral translates of the parallelepiped, where the translation vectors are precisely the elements of the discrete cone. In terms of the Minkowski sum, this can be written simply as:

$$
\operatorname{cone}_{\mathbb{R}}\left(a_{1}, \ldots, a_{n}\right)=\operatorname{cone}_{\mathbb{Z}}\left(a_{1}, \ldots, a_{n}\right)+\Pi\left(a_{1}, \ldots, a_{n}\right) .
$$

In particular

$$
\mathbb{Z}^{d} \cap \operatorname{cone}_{\mathbb{R}}\left(a_{1}, \ldots, a_{n}\right)=\operatorname{cone}_{\mathbb{Z}}\left(a_{1}, \ldots, a_{n}\right)+\left(\mathbb{Z}^{d} \cap \Pi\left(a_{1}, \ldots, a_{n}\right)\right) .
$$


This can be phrased in terms of multivariate generating functions. Consider the ring of generating functions in the variables $z_{1}, \ldots, z_{d}$ and write $z^{x}=z_{1}^{x_{1}} \cdot \ldots \cdot z_{d}^{x_{d}}$ for any integer point $x \in \mathbb{Z}^{d}$. Then

$$
\sum_{x \in \mathbb{Z}^{d} \cap \operatorname{cone}_{\mathbb{R}}\left(a_{1}, \ldots, a_{n}\right)} z^{x}=\frac{\sum_{x \in \mathbb{Z}^{d} \cap \Pi\left(a_{1}, \ldots, a_{n}\right)} z^{x}}{\left(1-z^{a_{1}}\right) \cdot \ldots \cdot\left(1-z^{a_{n}}\right)}
$$

since $\frac{1}{\left(1-z^{a_{1}}\right) \cdot \ldots \cdot\left(1-z^{a_{n}}\right)}$ is the multivariate generating function of cone $\mathbb{Z}^{d}\left(a_{1}, \ldots, a_{n}\right)$. Note that the numerator is a finite sum, so that if all of the components of the $a_{i}$ are nonnegative, the numerator is in fact a polynomial.

Now, let $n<d$ be integers and let $v_{1}, \ldots, v_{n} \in \mathbb{Z}_{\geqslant 0}^{d-1}$ denote the vertices of an integral simplex $\Delta$ in $\mathbb{R}^{d-1}$. By embedding $\Delta$ into $\mathbb{R}^{d}$ at height $x_{d}=1$, we pass to the vectors $a_{1}, \ldots, a_{n}$ with $a_{i}=\left(v_{i, 1}, \ldots, v_{i, d-1}, 1\right)$ and

$$
\#\left(\mathbb{Z}^{d-1} \cap k \Delta\right)=\#\left(\mathbb{Z}^{d} \cap \operatorname{cone}_{\mathbb{R}}\left(a_{1}, \ldots, a_{n}\right) \cap\left\{x \in \mathbb{R}^{d} \mid x_{d}=k\right\}\right)
$$

which, expressed in terms of generating functions, reads

$$
\sum_{k \geqslant 0} \sum_{x \in \mathbb{Z}^{d-1} \cap k \Delta} z_{1}^{x_{1}} \cdots z_{d-1}^{x_{d-1}} z_{d}^{k}=\sum_{x \in \mathbb{Z}^{d} \cap \operatorname{cone}_{\mathbb{R}}\left(a_{1}, \ldots, a_{n}\right)} z^{x}
$$

Combining identities (3) and (4), substituting 1 for $z_{1}, \ldots, z_{d-1}$ and substituting $z$ for $z_{d}$ we obtain

$$
\sum_{k \geqslant 0} L_{\Delta}(k) z^{k}=\frac{\sum_{i=0}^{n-1} h_{i}^{*} z^{i}}{(1-z)^{n}}
$$

where $h_{i}^{*}$ is the number of lattice points $x \in \Pi\left(a_{1}, \ldots, a_{n}\right)$ with $x_{d}=i$.

This completes the proof of Ehrhart's classic interpretation of the $h^{*}$-vector.

Theorem 11 (Ehrhart $[10,11])$. Let $v_{1}, \ldots, v_{n+1} \in \mathbb{Z}^{d}$ be linearly independent and let $a_{i}=\left(v_{i, 1}, \ldots, v_{i, d}, 1\right)$ for $i=1, \ldots, n+1$. Let $h^{*}=\left(h_{0}^{*}, \ldots, h_{n}^{*}\right)$ denote the $h^{*}$-vector of the $n$-dimensional simplex $\Delta=\operatorname{conv}\left(v_{1}, \ldots, v_{n+1}\right)$. Then

$$
h_{i}^{*}=\#\left(\mathbb{Z}^{d+1} \cap \Pi\left(a_{1}, \ldots, a_{n+1}\right) \cap\left\{x \in \mathbb{R}^{d+1} \mid x_{d+1}=i\right\}\right) .
$$

By virtue of the fact that polytopes are convex, the fact that every integral polytope can be triangulated and using a clever irrational shifting argument to get rid of lattice points on lower-dimensional faces [2], this theorem can be extended to general lattice polytopes.

Theorem 12 (Stanley [17]). Let $K$ be a d-dimensional integral polytope. Then the $h^{*}$ vector of $K$ is non-negative.

Our goal is now to obtain a similar counting interpretation, and, in particular, a similar non-negativity result for the $f^{*}$-vector of polytopal complexes. Before we come to this, we present examples of polytopal complexes where the $h^{*}$-vector has negative entries. 


\section{$2.6 \quad h^{*}$-vectors with negative entries}

Stanley's theorem tells us that in order to find $h^{*}$-vectors with negative entries we have to look outside the class of integral polytopes. We will consider integral polytopal complexes instead.

Coloring complexes of graphs are a class of simplicial complexes that have been studied by a number of authors in recent years, see, e.g., [7, 9, 12, 13, 14, 20]. All coloring complexes of graphs have a non-negative $h^{*}$-vector. A natural generalization are coloring complexes of hypergraphs. For details about this notion and about the computation of their $h$ - and $h^{*}$-vectors, we refer the interested reader to [7].

A hypergraph $H$ is a finite set $V$ of vertices, together with a set $E$ of edges. An edge is a set of vertices of cardinality at least two. A proper coloring of $H$ is a labeling $c$ of the vertices of $H$ with the property that every edge $e \in E$ contains at least two vertices $i, j \in e$ that have a different color $c_{i} \neq c_{j}$. Let $S$ be the set of all vectors in $x \in\{0,1\}^{|V|}$ that are not equal to the all-one and all-zero vectors. We can now define the simplicial complex $K$ which is called the coloring complex of $H$ as follows. $\sigma$ is a face of $K$ if and only if 1$) \sigma \subset S, 2)$ for any two vertices $x, y \in \sigma$ we have $x \leqslant y$ or $y \leqslant x$ componentwise and 3) there exists an edge $e \in E$ such that for all vertices $x \in \sigma$ and all $i, j \in e$ we have $x_{i}=x_{j}$. Notice that an element of $x \in S$ appears as a vertex of $K$ if and only if $x$, viewed as a coloring of the vertices of $H$ with exactly two colors 0 and 1 , is an improper coloring.

As an example, we consider the hypergraph $H$ on vertex set $\{1, \ldots, 10\}$ with edges $\{1,2,3,4,5,6\},\{4,5,6,7,8,9\}$ and $\{1,2,3,7,8,9\}$. The associated coloring complex $K$ is 3-dimensional. It consists of three 3 -dimensional spheres $S_{1}, S_{2}, S_{3}$ that intersect in a single common 0-dimensional subsphere $S^{\prime}$. The spheres $S_{i}$ all have the same combinatorial structure: They are each isomorphic to the simplicial complex obtained by taking the boundary complex of the 5 -dimensional cube $[0,1]^{5}$ triangulated by the braid arrangement and removing the all-zero and all-one vertices (and all incident faces).

Computation, by hand or using software packages such as Sage [19], shows that the $S_{i}$ have $h^{*}$-vector $h^{*}\left(S_{i}, 3\right)=(0,30,60,30)$. Similarly one can work out that the 3 dimensional $h^{*}$-vector of the 0 -dimensional sphere $S^{\prime}$, which consists of just two isolated vertices, is $h^{*}\left(S^{\prime}, 3\right)=(2,-6,6,-2)$. Given this data, we can then compute the $h^{*}$-vector of $K$ using inclusion-exclusion: After adding the $h^{*}$-vectors of the $S_{i}$ we have counted the common subsphere $S^{\prime}$ three times, so we have to subtract it twice to obtain the $h^{*}$-vector of $K$. Then, the $h^{*}$-vector of $K$ is

$$
\begin{aligned}
h^{*}(K, 3)=h^{*}\left(L_{K}, 3\right) & =h^{*}\left(L_{S_{1}}+L_{S_{2}}+L_{S_{3}}-2 L_{S^{\prime}}, 3\right) \\
& =3 h^{*}\left(S_{1}, 3\right)-2 h^{*}\left(S^{\prime}, 3\right) \\
& =3 \cdot(0,30,60,30)-2 \cdot(2,-6,6,-2) \\
& =(-4,102,168,94)
\end{aligned}
$$

which has a negative entry.

Intuitively speaking, the reason for the negative entry is that the complex consists of spheres that have an intersection of codimension strictly greater than 1. Further examples of hypergraph coloring complexes with negative entries in their $h^{*}$-vector can be 
constructed in this way.

\section{Partitioning a simplicial cone into discrete cones}

As we have seen, (2) gives a partition of the set of lattice points in $C=\operatorname{cone}_{\mathbb{R}}\left(a_{1}, \ldots, a_{n}\right)$ into discrete cones. This partition is ideally suited for the analysis of the $h^{*}$-vector. To get our hands on the $f^{*}$-vector, however, we need a different partition, given in Theorem 4 , which we will develop in this section. Whereas in (2) we used one translate of the fulldimensional discrete cone for each lattice point in the fundamental parallelepiped of $C$ to partition $\mathbb{Z}^{d} \cap C$, we will now use only atomic lattice points in the fundamental simplex of $C$ and the dimension of the discrete cone with an atomic lattice point $z$ as apex will depend on the height of $z$ in $C$.

The basic idea for this construction is illustrated in Figure 1. Consulting Figure 1 before reading this section is highly recommended.

Theorem 4 is the main technical result of this article, as the counting results in subsequent sections can be derived from Theorem 4 in a straightforward fashion. In order to prove this partition result, we first need a couple of definitions and an auxiliary lemma.

For every real number $x$ there exist an integer $\operatorname{int}(x) \in \mathbb{Z}$ and a real number $\operatorname{frac}(x) \in$ $(0,1]$ such that

$$
x=\operatorname{int}(x)+\operatorname{frac}(x) .
$$

Note that if $x$ is not an integer then $\operatorname{int}(x)=\lfloor x\rfloor$ and $\operatorname{frac}(x)=x-\lfloor x\rfloor$. But if $x \in \mathbb{Z}$, then $\operatorname{int}(x)=\lfloor x\rfloor+1$ and $\operatorname{frac}(x)=x-\lfloor x\rfloor-1$. So we call $\operatorname{int}(x)$ and $\operatorname{frac}(x)$ the skew integral and skew fractional part of $x$, respectively. If $v \in \mathbb{R}^{d}$ is a vector, we use int $(v)$ and $\operatorname{frac}(v)$ to denote the vector of skew integral and skew fractional parts of the components of $v$, respectively.

Given linearly independent integer vectors $v_{1}, \ldots, v_{d} \in \mathbb{Z}^{d}$, we define the fundamental simplex $\Delta\left(v_{1}, \ldots, v_{d}\right)$ generated by these vectors by

$$
\Delta\left(v_{1}, \ldots, v_{d}\right)=\left\{\sum_{i=1}^{d} \lambda_{i} v_{i} \mid 0 \leqslant \lambda_{i} \in \mathbb{R}, \sum_{i=1}^{d} \lambda_{i} \leqslant d\right\} .
$$

The half-open fundamental simplex is

$$
\Delta^{\circ}\left(v_{1}, \ldots, v_{d}\right)=\left\{\sum_{i=1}^{d} \lambda_{i} v_{i} \mid 0<\lambda_{i} \in \mathbb{R}, \sum_{i=1}^{d} \lambda_{i} \leqslant d\right\} .
$$

We say a point $z \in \operatorname{cone}_{\mathbb{R}}\left(v_{1}, \ldots, v_{d}\right)$ is at level $k$ if $z=\sum_{i=1}^{d} \lambda_{i} v_{i}$ with $k-1<$ $\sum_{i=1}^{d} \lambda_{i} \leqslant k$ and define $k=\operatorname{lev}(z)$ to be the level of $z$. We denote by $\operatorname{Lev}(k)$ the set of all lattice points in $\Delta^{\circ}\left(v_{1}, \ldots, v_{d}\right)$ at level $k$. 


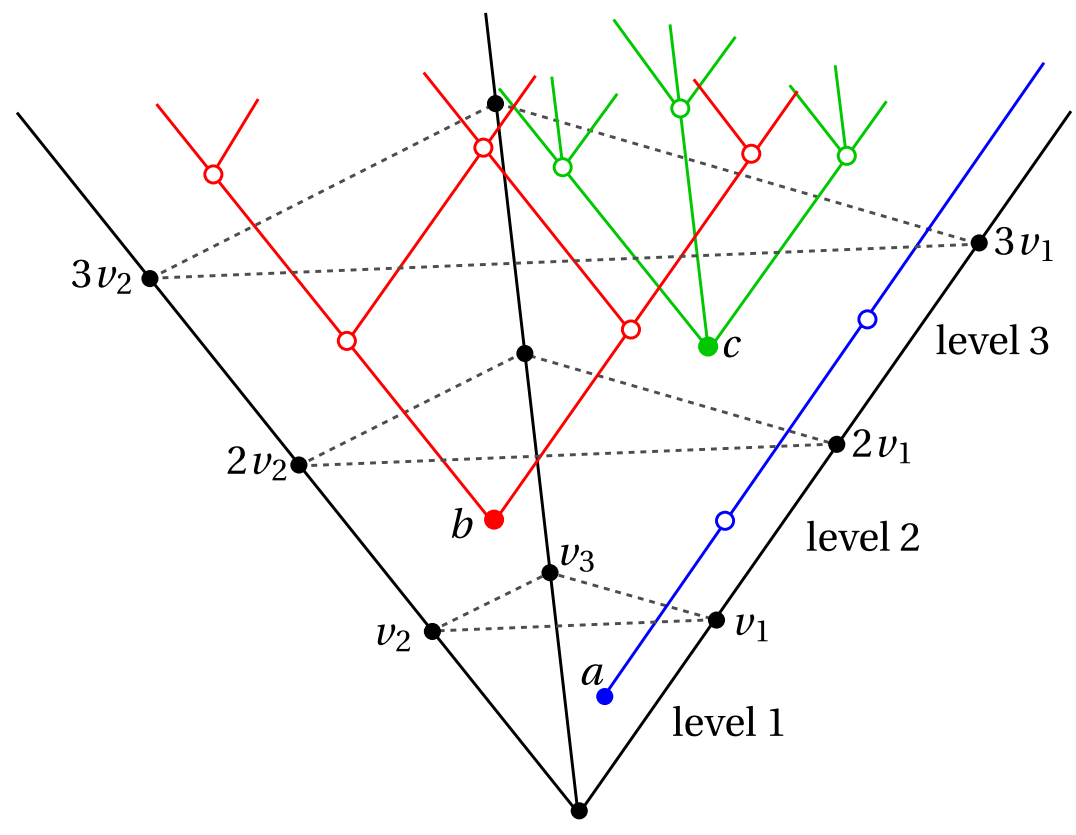

Figure 1: This figure illustrates Theorem 4. (Note that some lattice points in the cone are not shown here.) Suppose we have three linearly independent vectors $v_{1}, v_{2}, v_{3} \in \mathbb{R}^{3}$. The simplex conv $\left(3 v_{1}, 3 v_{2}, 3 v_{3}, 0\right)$ is the fundamental simplex of the cone generated by $v_{1}, v_{2}, v_{3}$. The half-open fundamental simplex is the intersection of the fundamental simplex with the interior of the cone. It is partitioned into three levels as indicated. The first level consists of all points below the hyperplane spanned by $v_{1}, v_{2}, v_{3}$, including the points on the hyperplane. The second level contains all points between the hyperplanes spanned by $v_{1}, v_{2}, v_{3}$ and $2 v_{1}, 2 v_{2}, 2 v_{3}$, respectively, excluding the points on the former but including the points on the latter and similarly for level 3 . The set of atomic lattice points in the cone is defined inductively. In the figure, the solid points in the interior of the cone show atomic lattice points, whereas the empty circles show lattice points that are not atomic. All lattice points at level 1 in the open cone are atomic, as the point labeled $a$ in the figure. Because $a$ is atomic, none of the points $a+k v_{1}$ for $1 \leqslant k \in \mathbb{Z}$ are atomic. Suppose $b$ is a lattice point in level 2 that cannot be reached from any atomic lattice point in level 1 by adding $v_{1}$. Then $b$ is atomic and all points that can be reached from $b$ by adding $v_{1}$ or $v_{2}$ are not atomic. Similarly, for any atomic lattice point $c$ in level 3, all points that be reached by adding the first three generators to $c$ are not atomic. There are no atomic lattice points above level 3 . The statement of Theorem 4 is that by placing $i$-dimensional discrete cones at all atomic lattice points in level $i$ in this way, we obtain a partition of the set of lattice points in the interior of $\operatorname{cone}_{\mathbb{R}}\left(v_{1}, v_{2}, v_{3}\right)$. 
We now define sets $T_{1}, \ldots, T_{d}$ with the property that $T_{i} \subset \operatorname{Lev}(i)$. The definition is inductive:

$$
\begin{aligned}
& T_{1}=\operatorname{Lev}(1) \\
& T_{k}=\operatorname{Lev}(k) \backslash\left(\bigcup_{i=1}^{k-1} \bigcup_{z \in T_{i}} z+\operatorname{cone}_{\mathbb{Z}}\left(v_{1}, \ldots, v_{i}\right)\right)
\end{aligned}
$$

We call the lattice points in $\bigcup_{i=1}^{\infty} T_{i}$ atomic. If $z=\sum_{i=1}^{d} \lambda_{i} v_{i}=: V \lambda$ for some atomic $z$, then we also call $\lambda$ atomic. If furthermore $x=\sum_{i=1}^{d} \mu_{i} v_{i}$ then note that for all $k$ we have

$$
x \in z+\operatorname{cone}_{\mathbb{Z}}\left(v_{1}, \ldots, v_{k}\right) \quad \text { if and only if } \mu \in \lambda+\operatorname{cone}_{\mathbb{Z}}\left(e_{1}, \ldots, e_{k}\right),
$$

where the $e_{i}$ denote the standard unit vectors.

Similar to our definition of $\operatorname{lev}(z)$, we write $\operatorname{lev}(\lambda)$ to denote the level of $\lambda$, i.e., $\operatorname{lev}(\lambda)$ is the unique integer such that $\operatorname{lev}(\lambda)-1<\sum_{i=1}^{d} \lambda_{i} \leqslant \operatorname{lev}(\lambda)$.

We write $\operatorname{deg}(\lambda)$ to denote the degree of $\lambda$ : If there exists an index $1 \leqslant j \leqslant d$ such that $\lambda_{j}>1$, then $\operatorname{deg}(\lambda)$ is defined to be the smallest such index. If there is no such index, we let $\operatorname{deg}(\lambda):=d+1$.

So $\lambda \in \mathbb{R}_{>0}^{d}$ is atomic if and only if $V \lambda$ is integer and there does not exist a $\mu \in \mathbb{R}_{>0}^{d}$ such that $V \mu$ is integer, $\mu \neq \lambda$ and

$$
\lambda \in \mu+\text { cone }_{\mathbb{Z}}\left(e_{1}, \ldots, e_{\operatorname{lev}(\mu)}\right) .
$$

These definitions are illustrated in Figure 1.

Despite their inductive definition, it turns out that atomic coefficient vectors $\lambda$ have a simple characterization.

Lemma 13. Let $z=\sum_{i=1}^{n} \lambda_{i} v_{i}$ be a lattice point in the interior of $\operatorname{cone}_{\mathbb{R}}\left(v_{1}, \ldots, v_{d}\right)$. This means in particular that $\lambda \in$ cone $_{\mathbb{R}}\left(e_{1}, \ldots, e_{d}\right)$. Then:

1. If $\lambda$ is not atomic, then $\operatorname{deg}(\lambda)<\operatorname{lev}(\lambda)$.

2. If $\operatorname{lev}(\lambda)=1$, then $\operatorname{deg}(\lambda)=d+1$.

3. If $\operatorname{lev}(\lambda)>d$, then $\operatorname{deg}(\lambda) \leqslant d$.

4. If $\operatorname{deg}(\lambda)<\operatorname{lev}(\lambda)$, there exists an atomic $\mu$ such that $\lambda \in \mu+\operatorname{cone}_{\mathbb{Z}}\left(e_{1}, \ldots, e_{\operatorname{lev}(\mu)}\right)$.

5. If $\operatorname{deg}(\lambda)<\operatorname{lev}(\lambda)$, then $\lambda$ is not atomic.

6. If $\operatorname{lev}(\lambda)>d$, then $\lambda$ is not atomic. In particular, there are only finitely many atomic lattice points.

So in particular we have the following characterization of atomicity:

$\lambda$ is atomic if and only if $\lambda_{j} \leqslant 1$ for all indices $j<\operatorname{lev}(\lambda)$. 
Or equivalently:

$\lambda$ is atomic if and only if $\operatorname{deg}(\lambda) \geqslant \operatorname{lev}(\lambda)$.

Proof of Lemma 13. We proceed in several steps.

Part (1): If $\lambda$ is not atomic, then $\operatorname{deg}(\lambda)<\operatorname{lev}(\lambda)$.

We have to show that there exists an index $j<\operatorname{lev}(\lambda)$ such that $\lambda_{j}>1$. If $\lambda$ is not atomic, then there exists an atomic $\mu$ with $\operatorname{lev}(\mu)<\operatorname{lev}(\lambda)$ such that $\lambda \in \mu+$ cone $_{\mathbb{Z}}\left(e_{1}, \ldots, e_{\operatorname{lev}(\mu)}\right)$, i.e., there exists a non-negative integral vector $\delta \in \mathbb{Z}_{\geqslant 0}^{d} \backslash\{0\}$ such that $\lambda=\mu+\delta$ with $\delta_{i}=0$ for all $i>\operatorname{lev}(\mu)$. As $\lambda \neq \mu, \delta_{j} \geqslant 1$ for some $j \leqslant \operatorname{lev}(\mu)$. Thus $\lambda_{j}>\delta_{j} \geqslant 1$ and $\operatorname{deg}(\lambda) \leqslant j \leqslant \operatorname{lev}(\mu)<\operatorname{lev}(\lambda)$ as desired.

Part (2): If $\operatorname{lev}(\lambda)=1$, then $\operatorname{deg}(\lambda)=d+1$.

We have $0<\sum_{i} \lambda_{i} \leqslant 1$ and $\lambda_{i}>0$ for all $i$. Thus $\lambda_{i} \leqslant 1$ for all $i$.

Part (3): If $\operatorname{lev}(\lambda)>d$, then $\operatorname{deg}(\lambda) \leqslant d$.

If $\sum_{i} \lambda_{i}>d$, then, by the pigeonhole principle, there is an $i$ such that $\lambda_{i}>1$ whence $\operatorname{deg}(\lambda) \leqslant i \leqslant d$.

Part (4): If $\operatorname{deg}(\lambda)<\operatorname{lev}(\lambda)$, then there exists an atomic $\mu \neq \lambda$ such that $\lambda \in \mu+$ cone $_{\mathbb{Z}}\left(e_{1}, \ldots, e_{\operatorname{lev}(\mu)}\right)$.

Let $\operatorname{lev}(\lambda)=l$. Let $\lambda^{l}, \lambda^{l-1}, \ldots, \lambda^{k}$ be a sequence of coefficient vectors with $\operatorname{lev}\left(\lambda^{i}\right)=i$ constructed recursively as follows. We start with $\lambda^{l}=\lambda$. Given $\lambda^{i}$, we distinguish two cases.

i. If $\operatorname{deg}\left(\lambda^{i}\right)<\operatorname{lev}\left(\lambda^{i}\right)$, then we define the next element in our sequence as $\lambda^{i-1}=$ $\lambda^{i}-e_{\operatorname{deg}\left(\lambda^{i}\right)}$. In this case, $\operatorname{lev}\left(\lambda^{i-1}\right)=\operatorname{lev}\left(\lambda^{i}\right)-1=i-1$ and $\operatorname{deg}\left(\lambda^{i-1}\right) \geqslant \operatorname{deg}\left(\lambda^{i}\right)$. Note that $\operatorname{deg}\left(\lambda^{i}\right) \leqslant d$, because either $\operatorname{lev}\left(\lambda^{i}\right) \leqslant d$ and then $\operatorname{deg}\left(\lambda^{i}\right)<d$ by assumption or $\operatorname{lev}\left(\lambda^{i}\right)>d$ and then $\operatorname{deg}\left(\lambda^{i}\right) \leqslant d$ by part $(3)$.

ii. If $\operatorname{deg}\left(\lambda^{i}\right) \geqslant \operatorname{lev}\left(\lambda^{i}\right)$, then we stop and $\lambda^{k}=\lambda^{i}$ is the last element of our sequence. Note that $k \geqslant 1$, as $\operatorname{lev}(\lambda)=1$ implies that $\operatorname{deg}(\lambda)=d+1$ by part $(2)$.

By part (1), we know that $\lambda^{k}$ is atomic as $\operatorname{deg}\left(\lambda^{k}\right) \geqslant \operatorname{lev}\left(\lambda^{k}\right)$. By construction, we know that

$$
\operatorname{deg}\left(\lambda^{l}\right) \leqslant \operatorname{deg}\left(\lambda^{l-1}\right) \leqslant \cdots \leqslant \operatorname{deg}\left(\lambda^{k+1}\right)<\operatorname{lev}\left(\lambda^{k+1}\right)=\operatorname{lev}\left(\lambda^{k}\right)+1
$$

whence

$$
\lambda^{l}=\lambda^{k}+\sum_{i=k+1}^{l} e_{\operatorname{deg}\left(\lambda^{i}\right)}
$$

where $\operatorname{deg}\left(\lambda^{i}\right) \leqslant \operatorname{lev}\left(\lambda^{k}\right)$ for all $i=k+1, \ldots, l$ and so

$$
\lambda \in \lambda^{k}+\operatorname{cone}_{\mathbb{Z}}\left(e_{1}, \ldots, e_{\operatorname{lev}\left(\lambda^{k}\right)}\right)
$$


as desired. Note that $\mu:=\lambda^{k} \neq \lambda$ as $\operatorname{deg}(\lambda)<\operatorname{lev}(\lambda)$ but $\operatorname{deg}(\mu) \geqslant \operatorname{lev}(\mu)$. Note also that $\mu-\lambda$ is integral, so that $\sum_{i} \mu_{i} v_{i}$ is a lattice point.

Part (5): If $\operatorname{deg}(\lambda)<\operatorname{lev}(\lambda)$, then $\lambda$ is not atomic.

By part (4), it follows that $\lambda \in \mu+\operatorname{cone}_{\mathbb{Z}}\left(e_{1}, \ldots, e_{\operatorname{lev}(\mu)}\right)$ for $\mu \neq \lambda$, which means that $\lambda$ is not atomic.

Part (6): If $\operatorname{lev}(\lambda)>d$, then $\lambda$ is not atomic. In particular, there are only finitely many atomic lattice points.

If $\operatorname{lev}(\lambda)>d$, then $\operatorname{lev}(\lambda)>\operatorname{deg}(\lambda)$ by part (3) and so $\lambda$ is not atomic by part (5). Since every level contains only finitely many lattice points, it follows that the total number of atomic lattice points is finite.

After these preparations, we can now show Theorem 4, the partition theorem at the heart of this article.

Theorem 4. Let $v_{1}, \ldots, v_{d}$ be linearly independent integer vectors in $\mathbb{Z}^{n}$ for $n \geqslant d$. Then

$$
\operatorname{relint}\left(\operatorname{cone}_{\mathbb{R}}\left(v_{1}, \ldots, v_{d}\right)\right) \cap \mathbb{Z}^{n}=\bigcup_{z \text { atomic }} z+\operatorname{cone}_{\mathbb{Z}}\left(v_{1}, \ldots, v_{\operatorname{lev}(z)}\right),
$$

where the union ranges over all atomic lattice points in the half-open fundamental simplex of $\operatorname{cone}_{\mathbb{R}}\left(v_{1}, \ldots, v_{d}\right)$ and this union is disjoint.

Proof of Theorem 4. First, we note that without loss of generality, we can assume $n=d$. Next, we observe that the right-hand side is contained in the left-hand side of (5) by construction. So we only have to show that the left-hand side is contained in the righthand side and that the union is disjoint.

The union is disjoint.

Let $\lambda=\alpha+\delta=\beta+\gamma$ where $\alpha$ and $\beta$ are atomic, $\delta \in \operatorname{cone}_{\mathbb{Z}}\left(e_{1}, \ldots, e_{\operatorname{lev}(\alpha)}\right)$ and $\gamma \in \operatorname{cone}_{\mathbb{Z}}\left(e_{1}, \ldots, e_{\operatorname{lev}(\beta)}\right)$. Without loss of generality, we assume that $\operatorname{lev}(\alpha) \leqslant \operatorname{lev}(\beta)$.

Note that because $\delta$ and $\gamma$ are integer vectors, $\operatorname{frac}(\lambda)=\operatorname{frac}(\alpha)=\operatorname{frac}(\beta)$ and, as both $\alpha$ and $\beta$ are atomic, $\alpha_{i}=\operatorname{frac}\left(\alpha_{i}\right)$ for all $i<\operatorname{lev}(\alpha)$ and $\beta_{i}=\operatorname{frac}\left(\beta_{i}\right)$ for all $i<\operatorname{lev}(\beta)$, by the characterization of atomicity. Furthermore,

- $\alpha_{i}=\beta_{i}$ for all $i<\operatorname{lev}(\alpha)$ because at these indices both $\alpha$ and $\beta$ are fractional, and - $\alpha_{i}=\beta_{i}$ for all $i>\operatorname{lev}(\beta)$ because at these indices $\alpha_{i}=\beta_{i}=\lambda_{i}$ by construction.

Now we distinguish two cases.

Case 1: $\operatorname{lev}(\alpha)<\operatorname{lev}(\beta)$.

- $\alpha_{i} \geqslant \beta_{i}$ for $\operatorname{lev}(\alpha) \leqslant i<\operatorname{lev}(\beta)$, because $\beta$ is fractional at these indices, and

- $\alpha_{i} \geqslant \beta_{i}$ for $i=\operatorname{lev}(\beta)$, because $\beta_{i} \leqslant \lambda_{i}=\alpha_{i}$. 
So $\alpha \geqslant \beta$ which implies $\operatorname{lev}(\alpha) \geqslant \operatorname{lev}(\beta)$, which gives a contradiction.

Case 2: $\operatorname{lev}(\alpha)=\operatorname{lev}(\beta)$.

In this case, we know $\alpha_{j}=\beta_{j}$ for all $j \neq \operatorname{lev}(\alpha)=\operatorname{lev}(\beta)$. So let $i=\operatorname{lev}(\alpha)$. First we observe that

$$
\lambda_{i}=\alpha_{i}+\delta_{i}=\beta_{i}+\gamma_{i}
$$

where $\delta_{i}$ and $\gamma_{i}$ are integers, so that $\alpha_{i}-\beta_{i}$ is an integer. Second we argue that because $\operatorname{lev}(\alpha)=\operatorname{lev}(\beta)$

$$
\left|\alpha_{i}-\beta_{i}\right|=\left|\sum_{j} \alpha_{j}-\sum_{j} \beta_{j}\right|<1 .
$$

Taking these observations together, we obtain $\alpha_{i}=\beta_{i}$ and hence $\alpha=\beta$, as desired.

The left-hand side of (5) is contained in the right-hand side.

Let $\lambda$ be the coefficient vector of a lattice point in the cone. If $\operatorname{deg}(\lambda) \geqslant \operatorname{lev}(\lambda)$, then $\lambda$ is atomic and hence contained in the right-hand side. Otherwise $\operatorname{deg}(\lambda)<\operatorname{lev}(\lambda)$ and thus, by part (4) of Lemma 13, there exists an atomic $\mu$ such that $\lambda=\mu+\operatorname{cone}_{\mathbb{Z}}\left(e_{1}, \ldots, e_{\operatorname{lev}(\mu)}\right)$, which shows that $\lambda$ is contained in the right-hand side as well.

\section{What $f^{*}$-vectors count}

We now apply the partition theorem from the previous section to obtain results on the $f^{*}$ vector of polytopes. We start out with the proof of Theorem 1, the counting interpretation of the $f^{*}$-coefficients of a lattice simplex.

Theorem 1. Let $\Delta \subset \mathbb{R}^{n}$ be an open lattice simplex, let $d^{\prime} \geqslant d=\operatorname{dim}(\Delta)$ and let $f^{*}\left(L_{\Delta}, d^{\prime}\right)=\left(f_{0}^{*}, \ldots, f_{d^{\prime}}^{*}\right)$. Then $f_{i}^{*}$ counts the number of atomic lattice points in the half-open fundamental simplex of $\operatorname{cone}_{\mathbb{R}}(\Delta \times\{1\})$ at level $i+1$.

Proof of Theorem 1. Let the vertices of $\Delta$ be denoted by $v_{1}, \ldots, v_{d+1}$. Then the vertices of $\Delta \times\{1\} \subset \mathbb{R}^{n+1}$ are linearly independent integer vectors $a_{1}, \ldots, a_{d+1} \in \mathbb{Z}^{n+1}$ with $a_{i}=\left(v_{i, 1}, \ldots, v_{i, n}, 1\right)$. Let

$$
C=\text { cone }_{\mathbb{R}}\left(a_{1}, \ldots, a_{d+1}\right)
$$

denote the cone generated by the $a_{i}$. As $\Delta$ is open, the number of lattice points in the $k$-th dilate of $\Delta$ equals the number of lattice points in the relative interior of $C$ at height $k$, i.e.,

$$
L_{\Delta}(k)=\#\left(\mathbb{Z}^{n+1} \cap \operatorname{relint}(C) \cap\left\{x \in \mathbb{R}^{n+1} \mid x_{n+1}=k\right\}\right) .
$$

By Theorem 4, we know

$$
\operatorname{relint}(C) \cap \mathbb{Z}^{n+1}=\bigcup_{z \text { atomic }} z+\operatorname{cone}_{\mathbb{Z}}\left(a_{1}, \ldots, a_{\operatorname{lev}(z)}\right)
$$


where the union is disjoint and runs over all atomic lattice points $z \in C$. As all $a_{i}$ have last coordinate equal to 1 and the number of non-negative integer solutions to $\lambda_{1}+\ldots+\lambda_{l}=k$ is $\left(\begin{array}{c}k+l-1 \\ l-1\end{array}\right)$, we have for any $1 \leqslant l \leqslant d+1$ and all integers $k \geqslant-l+1$ the identity

$$
\#\left(\mathbb{Z}^{n+1} \cap \operatorname{cone}_{\mathbb{Z}}\left(a_{1}, \ldots, a_{l}\right) \cap\left\{x \in \mathbb{R}^{n+1} \mid x_{n+1}=k\right\}\right)=\left(\begin{array}{c}
k+l-1 \\
l-1
\end{array}\right) .
$$

Note that the expression on the right-hand side of the above equation is a polynomial of degree $l-1$ in $k$. This polynomial evaluates to zero for $k \in\{-1, \ldots,-l+1\}$ and so the above identity holds for all integers $k \geqslant-l+1$. Let $z \in \mathbb{Z}^{n+1}$ be an integer vector with $z_{n+1}=l$. Then, translating the discrete cone on the left-hand side by $z$ amounts to shifting the counting polynomial by $l$. More precisely,

$$
\begin{aligned}
\#\left(\mathbb{Z}^{n+1} \cap\left(z+\operatorname{cone}_{\mathbb{Z}}\left(a_{1}, \ldots, a_{l}\right)\right) \cap\left\{x \in \mathbb{R}^{n+1} \mid x_{n+1}=k\right\}\right) & = \\
\#\left(\mathbb{Z}^{n+1} \cap \operatorname{cone}_{\mathbb{Z}}\left(a_{1}, \ldots, a_{l}\right) \cap\left\{x \in \mathbb{R}^{n+1} \mid x_{n+1}=k-l\right\}\right) & =\left(\begin{array}{c}
k-1 \\
l-1
\end{array}\right),
\end{aligned}
$$

for any $k \geqslant 1$. In particular, for any atomic lattice point $z \in C$ we have $z_{n+1}=\operatorname{lev}(z)$ and so

$$
\#\left(\mathbb{Z}^{n+1} \cap\left(z+\operatorname{cone}_{\mathbb{Z}}\left(a_{1}, \ldots, a_{\operatorname{lev}(z)}\right)\right) \cap\left\{x \in \mathbb{R}^{n+1} \mid x_{n+1}=k\right\}\right)=\left(\begin{array}{c}
k-1 \\
\operatorname{lev}(z)-1
\end{array}\right)
$$

for any integer $k \geqslant 1$. Combining (6), (7) and (8), we obtain

$$
L_{\Delta}(k)=\sum_{i=0}^{d}(\# \text { atomic } z \in C \text { at level } i+1) \cdot\left(\begin{array}{c}
k-1 \\
i
\end{array}\right)
$$

which proves the theorem.

The previous theorems allow us to prove Theorem 2, a complete characterization of $f^{*}$-vectors of integral partial polytopal complexes.

Theorem 2. A vector is the $f^{*}$-vector of some integral partial polytopal complex if and only if it is integral and non-negative.

Proof of Theorem 2. First, we argue that every integral partial polytopal complex has a non-negative $f^{*}$-vector. Let $K$ be an integral half-open polytopal complex of dimension $d$. Then the support of $K$ can be written as the disjoint union of relatively open lattice simplices $\sigma_{1}, \ldots, \sigma_{N}$, whence $f^{*}(K, d)$ is the sum of all $f^{*}\left(\sigma_{j}, d\right)$. By Theorem $1, f^{*}\left(\sigma_{j}, d\right)$ is a non-negative integer for all $j$ and hence, so is $f^{*}(K, d)$. Note that this argument only works because $f_{i}^{*}\left(\sigma_{j}, \operatorname{dim}\left(\sigma_{j}\right)\right)=f_{i}^{*}\left(\sigma_{j}, d\right)$ for all $0 \leqslant i \leqslant \operatorname{dim}\left(\sigma_{j}\right)$, a property the $h^{*}$-vector does not have.

Next, we argue that every non-negative integral vector $f^{*}=\left(f_{0}^{*}, \ldots, f_{d}^{*}\right)$ for some $d$ can be realized as the $f^{*}$-vector of some integral half-open polytopal complex $K$. This, however, is straightforward. Given $f^{*}$ we let $K$ be a polytopal complex that is the disjoint union of $f_{i}^{*}$ open unimodular $i$-dimensional lattice simplices for each $i=0, \ldots, d$. By construction $f^{*}(K, d)=f^{*}$, as desired. 
This implies in particular that every integral polytopal complex (and hence every lattice polytope) has a non-negative integral $f^{*}$-vector (Theorem 3 ). The crucial point here is that this holds even if the polytopal complex does not have a unimodular triangulation and even if its $h^{*}$-vector does have negative entries.

\section{The rational case}

Ehrhart's theorem for rational polytopes states that if $P$ is a rational polytope, then $L_{P}(k)$ is a quasipolynomial in $k$. A quasipolynomial is a function $q(k)$ such that there exists a number $m$ and polynomials $p_{0}(k), \ldots, p_{m-1}(k)$ such that $q(k m+l)=p_{l}(k)$ for all integers $k, l$ with $0 \leqslant l<m$. An $m$ with this property is called a period of the quasipolynomial $q$, whereas the minimal $m$ with this property is called the minimal period of $q$. Note that any positive integer $m$ such that $m P$ is an integral polytope is a period of $L_{P}$. The degree $\operatorname{deg}(q)$ of a quasipolynomial is the maximum degree of the polynomials $p_{i}$.

It is possible to define $h^{*}$-vectors for quasipolynomials and thus for rational polytopes and Stanley's non-negativity theorem also applies in this more general case $[1,2]$. In this section, we show how the above results for the $f^{*}$-vector can be generalized to the rational case.

For a given quasipolynomial $q$, a given period $m$ of $q$ and an integer $d \geqslant \operatorname{deg}(q)$ we define the $f^{*}$-vector of $q$ by

$$
\begin{aligned}
f^{*}(q, d, m)= & \left(f_{0}^{*}\left(p_{0}, d\right), \ldots, f_{0}^{*}\left(p_{m-1}, d\right),\right. \\
& f_{1}^{*}\left(p_{0}, d\right), \ldots, f_{1}^{*}\left(p_{m-1}, d\right), \\
& \ldots, \\
& \left.f_{d}^{*}\left(p_{0}, d\right), \ldots, f_{d}^{*}\left(p_{m-1}, d\right)\right) .
\end{aligned}
$$

Note that in this case

$$
q(k m+l)=\sum_{i=0}^{d} f_{i}^{*}\left(p_{l}, d\right) \cdot\left(\begin{array}{c}
k-1 \\
i
\end{array}\right),
$$

for all $0 \leqslant k, l \in \mathbb{Z}$ with $0 \leqslant l<m$ and $(k, l) \neq(0,0)$.

In analogy to the integral case, we define the $f^{*}$-vector of a rational polytope $P$ (or more generally a rational partial polytopal complex) to be $f^{*}\left(L_{P}, d, m\right)$ for a given $d \geqslant \operatorname{dim}(P)$ and a given period $m$ of $L_{P}$.

Given these conventions, we can now turn to the proof of Theorem 5.

Theorem 5. Let $\Delta \subset \mathbb{R}^{n}$ be an open rational simplex, let $d^{\prime} \geqslant d=\operatorname{dim}(\Delta)$ and $m$ be a positive integer such that $m \Delta$ is integral. There exist polynomials $p_{0}, \ldots, p_{m-1}$ such that for all integers $k \geqslant 0$ and $0 \leqslant l<m$ with $(k, l) \neq(0,0)$ the Ehrhart function of $\Delta$ satisfies $L_{\Delta}(k m+l)=p_{l}(k)$. Then for all $0 \leqslant i \leqslant d$ and all $0 \leqslant l<m$ the $f^{*}$-coefficient $f_{i}^{*}\left(p_{l}, d\right)$ counts the number of atomic lattice points $z$ in the half-open fundamental simplex of cone $_{\mathbb{R}}(\Delta \times\{m\})$ at level $i+1$ with $z_{n+1}=i m+l+1$. 
Proof of Theorem 5. The proof proceeds just as in the integral case, with the following differences. Let $v_{1}, \ldots, v_{d+1}$ denote the vertices of $\Delta$ and define generators $a_{1}, \ldots, a_{d+1}$ by $a_{i}=\left(m v_{i, 1}, \ldots, m v_{i, n}, m\right)$. Note that the vectors $a_{i}$ are integral by definition of $m$, but they are not primitive ${ }^{2}$ in general! We now consider the fundamental simplex of the cone $C=$ cone $_{\mathbb{R}}\left(a_{1}, \ldots, a_{d+1}\right)$ with respect to these generators and observe that the lattice points $z$ in $C$ at level $i+1$ have last coordinate $z_{n+1}=i m+l+1$ for some integral $0 \leqslant l<m$. All lattice points $z \in \operatorname{cone}_{\mathbb{Z}}\left(a_{1}, \ldots, a_{d+1}\right)$, however, have last coordinate $z_{n+1}=j m$ for some non-negative integer $j$. The theorem then follows.

Theorem 5 implies the non-negativity Theorem 6 just as in the integral case.

Theorem 6. Any rational partial polytopal complex has a non-negative integral $f^{*}$-vector.

Proof of Theorem 6 . Let $K$ be a rational partial polytopal complex of dimension $d=$ $\operatorname{dim}(P)$. Let $m$ be a positive integer such that $m K$ is integral. Let $T$ be a triangulation of $K$ that uses only vertices of $K$. By Theorem 5 all open simplices $\sigma$ in $T$ have an Ehrhart quasipolynomial $L_{\sigma}$ with period $m$. Thus

$$
f^{*}(K, d, m)=\sum_{\sigma \in T} f^{*}(\sigma, d, m)
$$

which shows that $f^{*}(K, d, m)$ is non-negative and integral.

Interestingly, there is another variant of Theorem 5 that makes use of a different grading of the cone over $\Delta$.

We define the restricted partition function $p_{m_{1}, \ldots, m_{d}}(k)$ of positive integers $m_{1}, \ldots, m_{d}, k$ to be the coefficient of $z^{k}$ in the Laurent expansion of the generating function

$$
\frac{1}{(1-z)^{m_{1}} \cdot \ldots \cdot(1-z)^{m_{d}}},
$$

or, equivalently,

$$
F_{m_{1}, \ldots, m_{d}}(k)=\#\left\{\left(\lambda_{1}, \ldots, \lambda_{d}\right) \mid 0 \leqslant \lambda_{i} \in \mathbb{Z}, \sum_{i=1}^{d} \lambda_{i} m_{i}=k\right\} .
$$

Theorem 7. Let $\Delta \subset \mathbb{R}^{n}$ be an open lattice simplex with vertices $v_{1}, \ldots, v_{d+1}$ and let $m_{1}, \ldots, m_{d+1}$ be minimal positive integers such that $m_{i} v_{i}$ is integral for all $1 \leqslant i \leqslant d+1$. Then

$$
L_{\Delta}(k)=\sum_{i=0}^{d} \sum_{s=0}^{S} c_{i, s} \cdot p_{m_{1}, \ldots, m_{i+1}}(k-s)
$$

for all $1 \leqslant k$ where $S=\sum_{i=1}^{d+1} m_{i}$ and $c_{i, s}$ denotes the number of atomic lattice points $z$ at level $i+1$ in the fundamental simplex of $\operatorname{cone}_{\mathbb{R}}\left(a_{1}, \ldots, a_{d+1}\right)$ with $z_{n+1}=s$. Here, $a_{i}=\left(m_{i} v_{i, 1}, \ldots, m_{i} v_{i, n}, m_{i}\right)$ for all $i=1, \ldots, d+1$.

\footnotetext{
${ }^{2}$ An integral vector $z \in \mathbb{Z}^{n}$ is called primitive if the line segment from $z$ to the origin contains no lattice point except its end points. Equivalently, $z$ is primitive if its components have greatest common divisor 1 .
} 
Proof of Theorem 7. Theorem 7 follows from Theorem 4 using the observation that for any lattice point $y$ with $0 \leqslant y_{n+1}=s \in \mathbb{Z}$ the value $p_{m_{1}, \ldots, m_{i+1}}(k-s)$ of the restricted partition function equals the number of lattice points $z$ in $y+\operatorname{cone}_{\mathbb{Z}}\left(a_{1}, \ldots, a_{i+1}\right)$ with $z_{n+1}=k$.

Acknowledgements. I would like to thank Matthias Beck for several stimulating discussions about an early version of this paper. I would also like to thank the anonymous referee for their helpful comments. Finally, my thanks go the DFG for funding this research.

\section{References}

[1] Matthias Beck and Sinai Robins. Computing the Continuous Discretely: IntegerPoint Enumeration in Polyhedra. Springer, 2007.

[2] Matthias Beck and Frank Sottile. Irrational proofs for three theorems of Stanley. European Journal of Combinatorics, 28(1):403-409, January 2007.

[3] Matthias Beck and Thomas Zaslavsky. Inside-out polytopes. Adv. Math., 205(1):134$162,2006$.

[4] Matthias Beck and Thomas Zaslavsky. The number of nowhere-zero flows on graphs and signed graphs. J. Combin. Theory Ser. B, 96(6):901-918, 2006.

[5] Felix Breuer and Aaron Dall. Viewing counting polynomials as Hilbert functions via Ehrhart theory. In 22nd International Conference on Formal Power Series and Algebraic Combinatorics (FPSAC 2010), pages 413-424. DMTCS, 2010.

[6] Felix Breuer and Aaron Dall. Bounds on the Coefficients of Tension and Flow Polynomials. Journal of Algebraic Combinatorics, 33(3):465-482, 2011.

[7] Felix Breuer, Aaron Dall, and Martina Kubitzke. Hypergraph coloring complexes. Discrete Mathematics, 312:2407-2420, 2012.

[8] Felix Breuer and Raman Sanyal. Ehrhart theory, Modular flow reciprocity, and the Tutte polynomial. Mathematische Zeitschrift, 270(1-2):1-18, 2012.

[9] Sarah Crown. The homology of the cyclic coloring complex of simple graphs. Journal of Combinatorial Theory, Series A, 116(3):595-612, April 2009.

[10] Eugène Ehrhart. Sur les polyèdres rationnels homothétiques à $n$ dimensions. $C . R$. Acad. Sci. Paris, 254:616-618, 1962.

[11] Eugène Ehrhart. Polynômes arithmétiques et méthode des polyèdres en combinatoire. International Series of Numerical Mathematics. Birkhäuser Verlag, Basel, 1977.

[12] Patricia Hersh and Ed Swartz. Coloring complexes and arrangements. Journal of Algebraic Combinatorics, 27(2):205-214, July 2007.

[13] Axel Hultman. Link complexes of subspace arrangements. European Journal of Combinatorics, 28(3):781-790, April 2007. 
[14] Jakob Jonsson. The Topology of the Coloring Complex. Journal of Algebraic Combinatorics, 21(3):311-329, May 2005.

[15] Petr Lisoněk. Combinatorial families enumerated by quasi-polynomials. Journal of Combinatorial Theory, Series A, 114(4):619-630, May 2007.

[16] Alexander Schrijver. Theory of Linear and Integer Programming. John Wiley \& Sons, Inc., 1986.

[17] Richard P. Stanley. Decompositions of Rational Convex Polytopes. Annals of Discrete Mathematics1, 6:333-342, 1980.

[18] Alan Stapledon. Inequalities and Ehrhart $\delta$-Vectors. Transactions of the American Mathematical Society, 361:5615-5626, 2009.

[19] W. A. Stein et al. Sage Mathematics Software (Version 4.8.0). The Sage Development Team, 2012. http://www. sagemath.org.

[20] Einar Steingrímsson. The Coloring Ideal and Coloring Complex of a Graph. Journal of Algebraic Combinatorics, 14(1):73-84, 2001.

[21] Günter M. Ziegler. Lectures on Polytopes. Graduate Texts in Mathematics. Springer, 1995. 